\title{
Co-infecção entre leishmaniose visceral e ehrlichiose monocítica em cães de Cuiabá, Mato Grosso
}

\author{
Co-infection between visceral leishmaniasis and monocitic ehrlichiosis in dogs from Cuiabá, Mato Grosso \\ Valéria Régia Franco Sousa ${ }^{1} \&$ Arleana do Bom Parto Ferreira de Almeida ${ }^{1}$
}

\begin{abstract}
RESUMO
A leishmaniose é uma zoonose causada pela Leishmania (Leishmania) chagasi em crescente expansão no Brasil, tendo como importante reservatório no ambiente urbano, os cães. O objetivo deste trabalho foi avaliar sorologicamente 199 cães com suspeita clínica de ehrlichiose monocítica canina atendidos no HOVET-UFMT no período de maio de 2004 a julho de 2005, encontrando-se cinco cães reagentes para leishmaniose canina na imunofluorescência indireta. Destes, dois apresentaram mórulas de Ehrlichia canis no esfregaço sangüíneo, evidenciando possível co-infecção desses agentes em áreas endêmicas.

Descritores: Leishmania (Leishmania) chagasi, Ehrlichia canis, Cão, Sorologia.

ABSTRACT

Leishmaniasis is a zoonosis caused by Leishmania (Leishmania) chagasi in growing expansion in Brazil, with the major reservoir in the urban environment, the dogs. The objective of this study was to evaluate serological 199 dogs with clinical suspicion of canine monocytic ehrlichiosis assisted in the HOVET-UFMT in the period from May 2004 to July 2005 and found to be five dogs seropositive for canine leishmaniasis by indirect immunofluorescence. Of these two had morulae of Ehrlichia canis in the blood smear showing a possible co-infection of these agents in endemic areas.
\end{abstract}

Keywords: Leishmania (Leishmania) chagasi, Ehrlichia canis, Dog, Serology.

${ }^{1}$ Departamento de Clínica Médica Veterinária, Faculdade de Agronomia e Medicina Veterinária (FAMEV), Universidade Federal de Mato Grosso (UFMT), Cuiabá, MT/Brasil. CORRESPONDÊNCIA: V. R. F. Sousa [regia@ufmt.br; Fax: + (65) 3615-8664. 


\section{INTRODUÇÃO}

No Brasil, a leishmaniose visceral tem como agente etiológico a Leishmania (Leishmania) chagasi transmitida por dípteros das espécies Lutzomyia longipalpis e L. cruzi, sendo descrita em quatro regiões e, no Estado de Mato Grosso, em 34 dos 141 municípios [7,17,22].

Epidemiologicamente, a leishmaniose visceral vem se caracterizando pela urbanização sofrida nos últimos anos, em decorrência dos desmatamentos e êxodo rural. Os primeiros surtos evidenciando urbanização da doença ocorreram em Belo Horizonte, Araçatuba e Campo Grande [18]. Nesse contexto, o cão possui um importante papel como reservatório da doença, por apresentar intenso parasitismo cutâneo [26].

A doença no cão e no homem tem características clínicas similares e inespecíficas, como febre irregular, anemia, perda de peso e caquexia no estágio final. Os cães acometidos podem apresentar linfoadenomegalia, hepatoesplenomegalia, onicogrifose, dermatite furfurácea, úlceras cutâneas, lesões oculares, além de distúrbios hemorrágicos [6].

O diagnóstico da leishmaniose visceral canina pode ser feito com base nas características clínicas e por métodos parasitológicos, sorológicos e moleculares. A imunofluorescência indireta é uma das técnicas preconizadas em inquéritos epidemiológicos por ser de fácil execução, rápida e barata [1,18]. Já o diagnóstico diferencial deve incluir, além de doenças cutâneas, a ehrlichiose monocítica e babesiose canina, com características clínicas semelhantes [8].

Este trabalho objetivou avaliar sorologicamente a infecção por Leishmania (Leishmania) chagasi em cães com suspeita clínica de ehrlichiose monocítica atendidos no Hospital Veterinário da Universidade Federal de Mato Grosso.

\section{MATERIAIS E MÉTODOS}

No período de setembro a dezembro de 2007 , no Laboratório de Leishmanioses do Hospital Veterinário da Universidade Federal do Mato Grosso (UFMT), foram analisadas amostras séricas de 199 cães com suspeita clínica de ehrlichiose monocítica atendidos no intervalo de maio de 2004 a julho de 2005, no Setor de Clínica Médica do referido hospital, na cidade de Cuiabá ( $\left.15^{\circ} 35^{\prime} 46^{\prime \prime} \mathrm{S} ; 56^{\circ} 05^{\prime} 48^{\prime \prime} \mathrm{O}\right)$, Mato Grosso.
As amostras foram processadas por imunofluorescência indireta com kit comercial (Biomanguinhos ${ }^{\circledR}$ - FIOCRUZ), seguindo o protocolo do fabricante. As amostras foram consideradas reagentes quando demonstravam fluorescência na diluição de 1:40, visualizada no microscópio de epifluorescência (DM1000® - Leica).

Os dados dos cães foram obtidos a partir dos prontuários arquivados e, desta forma, pode-se analisar os aspectos relacionados à procedência, características clínicas [14] e laboratoriais[11], além da presença de co-infecção com Ehrlichia canis.

\section{RESULTADOS}

$\mathrm{Na}$ imunofluorescência indireta cinco cães apresentaram reação com título de anticorpos igual ou superior a 1:40. Destes, um apresentou título igual a 1:160, dois apresentaram títulos de 1:320 e os outros, $1: 640$.

Destes cães, três eram fêmeas e dois machos, sendo dois sem raça definida, dois da raça cocker spaniel e um dog alemão. Três cães tinham entre um a três anos e dois entre três a seis anos. Dos positivos, quatro eram residentes na cidade de Cuiabá e um proveniente de cidade vizinha, Chapada dos Guimarães.

De acordo com as características clínicas (Figura 1) dos cães sororreagentes, quatro encontravam-se sintomáticos e um oligossintomático [11].

Os achados hematológicos demonstraram que os cinco cães sororreagentes apresentavam anemia normocítica normocrômica e quatro, trombocitopenia. No leucograma não se observou preponderância de nenhuma célula específica, sendo encontrado leucopenia intensa em um cão, linfopenia em outro cão,

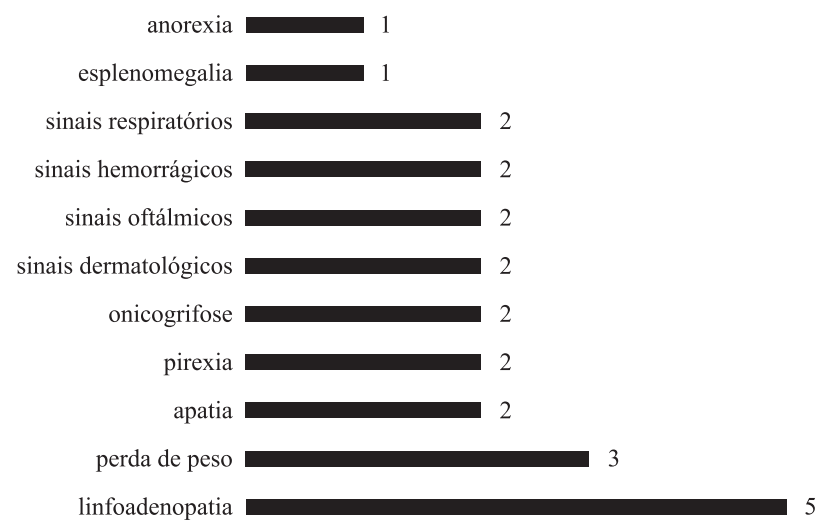

Figura 1. Principais sinais clínicos observados nos cães sororreagentes para leishmaniose visceral canina. 
monocitopenia em dois, neutropenia, eosinopenia e linfopenia em um cão cada, sendo que esses animais apresentavam a contagem total de células brancas totais dentro da normalidade.

A análise bioquímica dos cães revelou hiperproteinemia, com hipoalbuminemia e hiperglobulinemia em quatro cães. A avaliação hepática desses animais não demonstrou alteração na atividade sérica de alanino amino transferase, aspartato amino transferase e fosfatase alcalina, entretanto um cão apresentou azotemia, isto é, elevada concentração sérica de uréia e creatinina.

Dos cães com anticorpos para leishmaniose, dois apresentaram mórulas de Ehrlichia canis no esfregaço sangüíneo.

\section{DISCUSSÃO}

A prevalência estimada para o grupo estudado foi de $2,51 \%$, sendo inferior à média obtida na região metropolitana de Cuiabá no ano de 1999 [20]. De acordo com o Ministério da Saúde quando a prevalência canina for maior ou igual a $2 \%$, preconizase a realização de inquérito canino censitário [18]. Em levantamento sorológico canino realizado em municípios de Mato Grosso, no período de 1998-2005, a capital Cuiabá apresentou um percentual de 8,4\% dos cães positivos [17]. Entretanto, esses dados foram obtidos de inquéritos realizados em períodos distintos, não possuindo caráter censitário.

O encontro de quatro cães sororreagentes para leishmaniose, provenientes da cidade de Cuiabá, reforça o descrito na literatura da urbanização desta doença $[18,26]$. O primeiro relato de leishmaniose visceral humana em Cuiabá ocorreu em 2005 com registro de cinco casos [17]. Geograficamente, a capital de Mato Grosso localiza-se na região CentroSul do estado, caracterizada por apresentar grande mobilidade populacional, sendo banhada pelo rio Cuiabá e seu afluente rio Coxipó, apresentando mata ciliar e população ribeirinha significativa [23]. A crescente migração populacional aos centros urbanos associada à proximidade com rios e matas, o crescente desmatamento e ocupação desordenada aliada ao aumento da densidade do vetor e proximidade do homem com reservatório doméstico, tem caracterizado a ocorrência de casos de leishmaniose visceral em diversas cidades brasileiras, inclusive em Cuiabá [17$19,26]$.
Como preconizado pelo Ministério da Saúde, utilizou-se para a análise sorológica dos cães a reação de imunofluorescência indireta, método este considerado útil em inquéritos epidemiológicos da doença, por apresentar uma sensibilidade e especificidades adequadas contribuindo de forma segura na identificação de casos positivos, reduzindo a ocorrência de resultados falsos positivos e negativos $[1,13,18]$.

$\mathrm{Na}$ leishmaniose visceral canina não se observa predisposição sexual, racial, assim como não se verificou no grupo analisado [6,9]. Neste estudo, a observação dos cães infectados apenas na faixa etária adulta pode ser explicada pelo longo período de incubação do parasito que, em média, varia de três a seis meses até vários anos [15].

Os cães sororreagentes apresentaram-se sintomáticos e oligossintomáticos no momento da consulta. Dos sinais clínicos apresentados a linfadenopatia e emagrecimento progressivo foram os mais prevalentes, fato comumente observado nos cães com leishmaniose visceral e ehrlichiose crônica [6,16,25]. Observou-se uma relação direta entre gravidade sintomática e título de anticorpos nos cães sororreagentes.

Apesar de ser observado em $68 \%$ dos cães com leishmaniose visceral, as alterações dermatológicas foram observadas em apenas dois, no entanto, o baixo número de cães sororreagentes para a doença impossibilita análise estatística fidedigna [4].

Um predomínio de cães infectados com anemia normocítica normocrômica e aumento das proteínas plasmáticas totais é descrito na literatura para ambas as doenças, relacionando-se aos achados de cães sintomáticos, fato observado nos animais desta pesquisa $[2,24,28]$. A hiperproteinemia nos cães com leishmaniose é decorrente de uma resposta imune humoral policlonal, observando-se um aumento de gamaglobulina, diminuição de albumina e inversão na relação albumina globulina. Em quatro cães verificou-se a correlação entre a exacerbação dos sinais clínicos da leishmaniose visceral canina, aumento da proteína sérica, globulina e diminuição de albumina [10,24].

$\mathrm{O}$ encontro de cães com trombocitopenia na leishmaniose visceral tem sido descrito como decorrente de seqüestro esplênico e, ocasionalmente, por aplasia ou hipoplasia medular que, aliada à vasculite, uremia e insuficiência hepática levam a tendências hemorrágicas [6]. As alterações na quantidade e função plaquetária, indicando alteração na hemostasia primária, e na síntese e metabolismo dos fatores de 
coagulação, são mais evidentes nos cães sintomáticos devido ao comprometimento do sistema imune e processo inflamatório tanto na ehrlichiose quanto na leishmaniose canina, fato observado neste estudo $[3,5]$.

O encontro de mórula de Ehrlichia canis em dois cães sororreagentes para leishmaniose provoca o agravamento do quadro clínico, sendo comum em áreas endêmicas para ambas as doenças, fato encontrado nesta capital, onde se tem relatado um grande número de cães com ehrlichiose monocítica canina [6,27]. Alguns autores relatam a ocorrência de reação cruzada entre ehrlichiose e leishmaniose nos testes sorológicos, no entanto em estudos experimentais, observou-se a não ocorrência de tal reação $[8,12$, 21].

\section{CONCLUSÃO}

A similaridade clínica e a endemicidade entre leishmaniose visceral e ehrlichiose canina torna difícil o diagnóstico. Desta forma, conclui-se a importância de realizar exames complementares diferenciais, já que as duas possuem prognóstico e tratamento distintos.

Agradecimento. A Fundação de Amparo a Pesquisa do Estado de Mato Grosso (FAPEMAT) e Coordenação de Aperfeiçoamento de Pessoal de Nível Superior (CAPES) pelo apoio financeiro.

\section{REFERÊNCIAS}

1 Alves W.A. \& Bevilacqua P.D. 2004. Reflexões sobre a qualidade do diagnóstico da leishmaniose visceral canina em inquéritos epidemiológicos: o caso da epidemia de Belo Horizonte, Minas Gerais, Brasil, 1993-1997. Caderno de Saúde Pública. 20: 259-265.

2 Breitschwerdt E.B. 2004. Riquetsioses In: Ettinger S.J. \& Feldman E.C. (Eds). Tratado de medicina Interna Veterinária. Doenças do Cão e do Gato. 5.ed. Rio de Janeiro: Guanabara Koogan, pp. 422-229.

3 Bulla C., Takahira R.K., Araújo Jr. J.P., Trinca L.A., Lopes R.S. \& Wiedmeyer C.E. 2004. The relationship between the degree of thrombocytopenia and infection with Ehrlichia canis in an endemic area. Veterinary Research. 35: 141-146.

4 Cavalcanti M.P., Faustino M.A.G., Silva L.B.G. \& Alves L.C. 2005. Aspectos clínicos das dermatopatias infecciosas e parasitárias em cães com diagnóstico presuntivo de leishmaniose visceral. Clínica Veterinária. 58: 36-42.

5 Ciaramella P., Pelagalli A., Cortese L., Pero, M.E., Corona M., Lombardi P., Avallone, L. \& Persechino A. 2005. Altered platelet aggregation and coagulation disorders related to clinical findings in 30 dogs naturally infected by Leishmania infantum The Veterinary Journal. 169: 465-467.

6 Feitosa M.M., Ikeda F.A., Luvizotto M.C.R. \& Perri S.H.V. 2000. Aspectos clínicos de cães com leishmaniose visceral no município de Araçatuba, São Paulo (Brasil). Clínica Veterinária. 28: 36-44.

7 Galati E.A.B., Nunes V.L.B., Rego Jr. F.A.R., Oshiro E.T. \& Cang M.R. 1997. Estudo de flebotomíneos (Díptera: Psychodidae) em foco de leishmaniose visceral no Estado de Mato Grosso do Sul, Brasil. Revista de Saúde Pública. 31: 378-390.

8 Gomes Y.M., Cavalcanti M.P., Lira R.A., Abath, F.G.C. \& Alves L.C. 2006. Diagnosis of canine visceral leishmaniasis: Biotechnological advances. The Veterinary Journal. 175: 45-52.

9 Gontijo C.M.F. \& Melo M.N. 2004. Leishmaniose visceral no Brasil: quadro atual, desafios e perspectivas. Revista Brasileira de Epidemiologia. 7: 338-349.

10 Guillén Llera J.L., López García M.L., Martín Reinoso E. \& De Vivar González R. 2002. Differential serological testing by simultaneous indirect immunofluorescent antibody test in canine leishmaniosis and ehrlichiosis. Veterinary Parasitology. 109: 185-190.

11 Ikeda F.A., Ciarlini P.C., Feitosa M.M., Gonçalves M.E., Luvizotto M.C.R. \& Lima V.M.F. 2003. Perfil hematológico de cães naturalmente infectados por Leishmania chagasi no município de Araçatuba - SP: um estudo retrospectivo de 191 casos. Clínica Veterinária. 47: 42-48.

12 Kaneko J.J., Harvey J.W. \& Bruss M.L. 1997. Clinical biochemistry of domestic animals. 5th edn. San Diego: Academic Press, 932p.

13 Machado J.G., Moraes J.R.C., Costa R.T., Nascimento E. \& Moreira E.C. 2007. Comparação dos resultados dos métodos de imunofluorescência indireta e Elisa indireta no diagnóstico sorológico da leishmaniose visceral canina realizado pelos laboratórios de Belo Horizonte, MG, Brasil. Veterinária e Zootecnia. 14: 47-51.

14 Mancianti F., Gramiccia M., Gradoni L. \& Pieri S. 1998. Studies on canine leishmaniasis control. I. Evolution of infection of different clinical forms of canine leishmaniasis following antimonial treatment. Transactions of the Royal Society of Tropical Medicine and Hygiene. 82: 566-567. 
15 Marzochi M.C.A., Coutinho S.G., Sabroza P.C., Souza M.A., Souza P.P., Toledo L. M. \& Filho F.B.R. 1985. Leishmaniose visceral canina no Rio de Janeiro - Brasil. Caderno de Saúde Pública. 1: 432-446.

16 Mcdade J.E. 1990. Ehrlichiosis - a disease of animals and humans. Journal of Infectious Diseases. 161: 609-617.

17 Mestre G.L.C. \& Fontes C.J.F. 2007. A expansão da epidemia de leishmaniose visceral no Estado de Mato Grosso, 19982005. Revista da Sociedade Brasileira de Medicina Tropical. 40: 42-48.

18 Ministério da Saúde (MS). 2003. Secretaria de Vigilância em Saúde. Departamento de Vigilância Epidemiológica. Manual de Controle da Leishmaniose Visceral. Série A. Normas e Manuais Técnicos. Brasília, DF.

19 Monteiro É.M., Silva J.C.F., Costa R.T., Costa D.C., Barata R.A., Paula E.V., Machado-Coelho G.L.L., Rocha M.F., Fortes-Dias C.L. \& Dias E.S. 2005. Leishmaniose visceral: estudo de flebotomíneos e infecção canina em Montes Claros, Minas Gerais. Revista da Sociedade Brasileira de Medicina Tropical. 38: 147-152.

20 Moura S.T., Fernandes C.G.N., Pandolpho V.C. \& Silva R.R. 1999. Diagnóstico de leishmaniose canina na área urbana do município de Cuiabá, Estado de Mato Grosso, Brasil. Brazilian Journal of Veterinary Research and Animal Science. 36 : 101-102.

21 Oliveira T.M.F.S., Carvalho D., Furuta P.I. \& Machado R.Z. 2006. Estudo da reação cruzada entre Leishmania Chagasi, Ehrlichia Canis e Babesia Canis nos testes sorológicos de imunofluorescência indireta e ensaio imunoenzimático. In: Anais do $1^{\circ}$ Fórum sobre Leishmaniose Visceral Canina (Jaboticabal, Brasil), p.59.

22 Palatnik-de-Sousa C.B., Santos W.R., França-Silva J.C., Costa R.T., Reis A.B., Palatnik M.M., W. \& Genaro O. 2001. Impact of canine control on the epidemiology of canine and human visceral leishmaniasis in Brazil. American Journal Tropical Medicine and Hygiene. 65: 510-517.

23 Prefeitura Municipal de Cuiabá. 2004. Perfil Socioeconômico de Cuiabá, volume II. Cuiabá: Instituto de Pesquisa e Desenvolvimento Urbano, 407p.

24 Reis A.B., Martins-Filho O.A., Teixeira-Carvalho A., Carvalho M.G., Mayrink W., França-Silva J.C., Giunchetti R.C., Genaro O. \& Correâ-Oliveira R. 2006. Parasite density and impaired biochemical/hematological status are associated with severe clinical aspects of canine visceral leishmaniasis. Research in Veterinary Science. 81: 68-75.

25 Rikihisa Y. 1991. The tribe Ehrlichiae and ehrlichial diseases. Clinical Microciology Review. 4: 286-308.

26 Silva A.V.M., Paula A.A., Cabrera M.A.A. \& Carreira J.C.A. 2005. Leishmaniose em cães domésticos: aspectos epidemiológicos. Caderno de Saúde Pública. 21: 324-328.

27 Sousa V.R.F. 2006. Avaliação clínica, morfológica, hematológica, bioquímica e biomolecular de cães naturalmente infectados por Ehrlichia canis e Anaplasma platys. 46f. Seropédica, RJ. Tese (Doutorado em Ciências Veterinárias) - Programa de Pós-graduação em Ciências Veterinárias. Universidade Federal Rural do Rio de Janeiro.

28 Waner T. \& Harrus S. 2000. Canine monocytic ehrlichiosis (CME) In: Recent Advances in Canine Infectious Disease. 5p. Disponível em: <http://www.ivis.org>. Acessado em 02/2008.

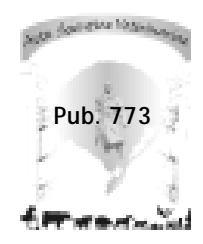

